\title{
Congenital coronary artery anomaly simulating an acute aortic dissection
}

\author{
L De Luca, F Bovenzi, I de Luca
}

Heart 2004;90:e1 1. (www.heartjnl.com/cgi/contents/full/90/3/e1 1). doi: 10.1136/hrt.2003.021337

In this rare case, a patient had an anomalous right coronary artery originating from the left sinus of Valsalva coursing between the great vessels. He presented with symptoms and computed tomograms suggestive of an acute aortic dissection.

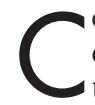
oronary artery anomalies (CAA) are difficult to diagnose on clinical grounds. According to the literature, the incidence of CAA is reported to be between $0.46-1.55 \%$ in the general population. ${ }^{1}$ The clinical presentations and functional importance of CAA vary widely. On the one extreme, they may be clinically silent, discovered by chance and considered only to be curiosities; on the other extreme, they may cause angina pectoris, syncope, arrhythmias, myocardial infarction, and even sudden cardiac death. Significant morbidity and mortality can result, even among young adults. The reason for the sudden fatal events is generally unclear but probably relates to the anatomical variants and proximal course of the coronary anomaly. In particular, anomalies that involve the proximal coronary artery segments coursing between the aorta and the pulmonary trunk have been reported to be potentially lethal. ${ }^{2}$

We describe, for the first time in the literature, a patient with an anomalous coronary artery with an aberrant course between the great vessels misdiagnosed as being an acute aortic dissection on the basis of the clinical presentation and computed tomography (CT).

\section{CASE REPORT}

A 43 year old man with a history of hypertension and hypercholesterolaemia presented to the emergency room of our hospital for sudden tearing pain in his left arm radiating to the back that began after he competed in an athletic event. His blood pressure was 145/70 mm Hg. His ECG pattern and cardiac markers were normal. A transthoracic echocardiogram showed mild dilatation of the aortic root $(44 \mathrm{~mm})$ and the aortic arch $(37 \mathrm{~mm})$, and a bicuspid aortic valve with a moderate stenosis and mild aortic regurgitation. A CT examination with intravenous injection of contrast was urgently performed. This study showed a $1 \mathrm{~cm}$ hypodense stripe suggestive of an aortic dissection on the posterior wall of the ascending aorta, immediately above the cardiac valvar plane (fig 1). Transoesophageal echocardiography (TOE) did not confirm the presence of an aortic dissection. Rather, it showed an anomalous course of a coronary artery between the aorta and right ventricular outflow tract (fig 2). Coronary angiography subsequently documented an anomalous right coronary artery arising from the left sinus of Valsalva. The ostium of the anomalous artery was separate from that of the left main artery (fig 3). No coronary stenoses were detected during coronary angiography.
The patient was discharged three days later in good clinical condition with the recommendation to return for corrective surgery.

\section{DISCUSSION}

Aortic dissection is a serious, life threatening condition that requires prompt diagnosis and treatment. The sensitivity and specificity for the detection of this disorder with CT are $94 \%$ and $87 \%$, respectively. ${ }^{3}$ Moreover, the ascending aorta is the most difficult region for $\mathrm{CT}$ to detect a dissection accurately because of significant motion artefacts.

TOE has many discrete advantages in the diagnosis of acute aortic dissection, the most important of which is its documented accuracy, with a reported sensitivity and specificity of $99 \%$ and $98 \%$, respectively. ${ }^{4}$ Visualisation of the ascending root is feasible in most patients who undergo TOE. TOE can accurately diagnose aortic dissections and can be a useful adjunct to CT, particularly when the ascending aorta is involved. The TOE of our patient assisted in deriving the correct diagnosis. TOE should therefore be considered for other patients who present with suspected aortic dissection, particularly if CT suggests pathology only in the aortic root, where erroneous readings on CT are most likely to occur.

Nowadays, TOE and CT are both imaging and screening methods recommended for detecting CAA. ${ }^{1}$ A detailed assessment of the coronary artery anatomy is an established part of the evaluation of structural heart defects, but it is being increasingly incorporated into the evaluation of chest pain and pre-athletic screens. In fact, CAA are usually compatible with normal myocardial development and postnatal function, even permitting normal athletic activity in most people. On the other hand, sudden death is frequently the only symptom of a coronary anomaly. ${ }^{1}$ According to the sudden death committee of the American Heart Association, ${ }^{5}$ CAA cause $19 \%$ of deaths in athletes. Moreover, Burke and colleagues $^{6}$ reported that CAA are involved in about $12 \%$ of sports related sudden cardiac deaths compared with $1.2 \%$ of non-sports related deaths in 14-40 year old patients.

Anomalous origins of the left main coronary artery from the right aortic sinus of Valsalva and of the right coronary artery from the left sinus of Valsalva are the most common CAA associated with angina pectoris, myocardial infarction, or sudden cardiac death. ${ }^{7}$ The incidence of morbidity and death is even higher when the coronary anomaly is associated with other anomalies such as a bicuspid aortic valve. ${ }^{8}$ In these cases, corrective surgery is recommended.

The pathophysiological mechanism most likely to explain chest pain and sudden death is that CAA are often associated with an anomalous course between the great vessels. Thus, the aorta and pulmonary artery impinge on the anomalous

Abbreviations: CAA, coronary artery anomalies; $C T$, computed tomography; TOE, transoesophageal echocardiography 


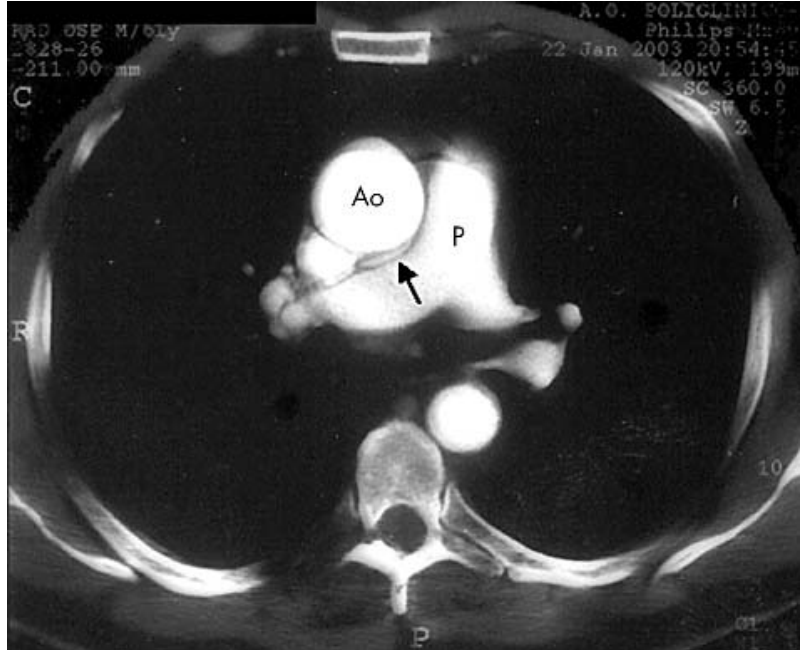

Figure 1 Computed tomography with intravenous injection of contrast agent. A hypodense stripe on the posterior wall of the ascending aorta (Ao) comparable with an aortic dissection is visible (arrow). P, Pulmonary artery.

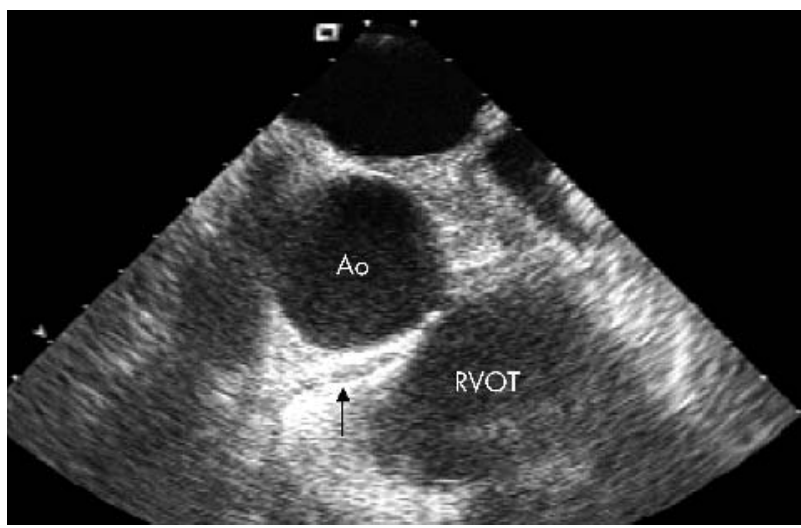

Figure 2 Transoesophageal echocardiography (basal view, short axis at $35^{\circ}$ ) detected an anomalous course of a coronary artery (arrow) between the aorta (Ao) and the right ventricular oufflow tract (RVOT).

vessel, especially during exercise when there is aortic and pulmonary artery dilatation, and this is coincident with increased coronary flow demand. ${ }^{1}$ Our patient's chest pain was likely a result of a similar sequence of events. Other imaging techniques have confirmed the presence of ischaemia in such patients. Recently, a perfusion cardiovascular magnetic resonance study ${ }^{9}$ documented a perfusion defect in a similar patient with CAA coursing between the aorta and pulmonary artery. We also previously observed reversible myocardial perfusion defects with stress/rest sestamibi 99technetium single photon emission computed tomography in 10 patients with angiographically documented CAA $^{10}$ with an anomalous course between the great vessels.

In conclusion, CAA should be taken into consideration in the clinical setting of chest pain and sudden death, even among young athletes. An integrated approach using CT and

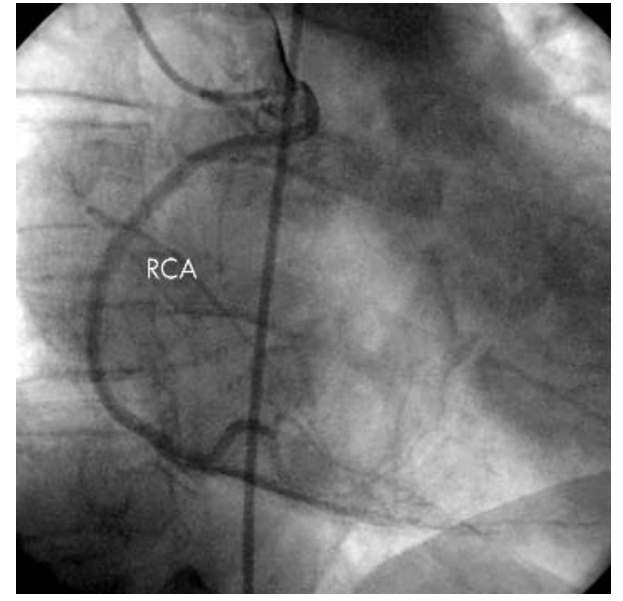

Figure 3 Coronary angiography (anteroposterior view) documented an anomalous origin of the right coronary artery (RCA), selectively engaged, from the left sinus of Valsalva.

TOE should be desirable for differential diagnosis between aortic dissection and proximal course of CAA.

\section{Authors' affiliations}

L De Luca, F Bovenzi, I de Luca, Department of Cardiology, Azienda Policlinico, Bari, Italy

Correspondence to: Dr L De Luca, Divisione Ospedaliera di Cardiologia-Azienda Policlinico, Piazza G Cesare, 11, 70100 Bari, Italy; leo.deluca@libero.it

Accepted 22 September 2003

\section{REFERENCES}

1 Angelini P, Velasco JA, Flamm S. Coronary anomalies: incidence, pathophysiology, and clinical relevance. Circulation 2002; 105:2449-54

2 Liberthson RR, Dinsmore RE, Fallon JT. Aberrant coronary artery origin from the aorta: report of 18 patients, review of literature and delineation of natural history and management. Circulation 1979:59:748-54.

3 Erbel R, Oelert H, Meyer J, et al. Effect of medical and surgical therapy on aortic dissection evaluated by transesophageal echocardiography: implication for prognosis and therapy. The European Cooperative Study Group on Echocardiography. Circulation 1993;88:111-10.

4 Erbel R, Engberding R, Daniel W, et al. Echocardiography in diagnosis of aortic dissection. Lancet 1989;i:457-61.

5 Maron BJ, Thompson PD, Puffer JC, et al. Cardiovascular preparticipation screening of competitive athletes: a statement for health professional from the sudden death committee (clinical cardiology) and congenital cardiac defects committee (cardiovascular disease in the young), American Heart Association. Circulation 1996;94:850-6

6 Burke AP, Farb A, Virmani R, et al. Sports-related and non-sports-related sudden cardiac deaths in young adults. Am Heart J 1991;121:568-75.

7 Kragel AH, Roberts WC. Anomalous origin of either the right or left main coronary artery from the aorta with subsequent coursing between aorta and pulmonary trunk: analysis of 32 necropsy cases. Am J Cardiol 1988;62:771-7.

8 Sans-Coma V, Duran AC, Fernandez B, et al. Coronary artery anomalies and bicuspid aortic valve. In: Angelini $\mathrm{P}$, ed. Coronary artery anomalies. Philadelphia: Lippincott Williams \& Wilkins, 1999:17-27.

9 Bunce NH, Rahman SL, Keegan J, et al. Anomalous coronary arteries: anatomic and functional assessment by coronary and perfusion cardiovascular magnetic resonance in three sisters. J Cardiovasc Magn Reson 2001;3:361-9.

10 Bovenzi F, Colonna P, De Luca L, et al. Coronary angiography, transesophageal echocardiography and stress-rest scintigraphy to evaluate the functional importance of anomalous coronary arteries in adults [abstract]. J Am Coll Cardiol 2002;39:290B. 